Pure Sciences

Poster

Abstract ID: 94

\title{
Patternable polymer microstructure for optical biosensing
}

\author{
Mohd Hazimin Mohd Salleh ${ }^{\mathrm{a}} \mid$ Mohd Haziq MS $^{\mathrm{b}} \mid$ Muhammad Salihi Abd Hadi ${ }^{\mathrm{a}}$ \\ ${ }^{a}$ Kulliyyah of Science, International Islamic University Malaysia \\ ${ }^{b}$ Kulliyyah of Information and Communication Technology, International Islamic University Malaysia
}

Introduction: Applications of rapid sensing and detection of biological analytes are growing due to the significant environmental monitoring, health screening, cell growth and bio/chemical sensors. These factors influence the research and development on simple, cheap and sensitive functional biomolecular device. The interest in the label-free optical detection has been increased as the optical waveguide has a direct light interaction with surrounding analytes, easy integration with microfluidic system and the capability to provide specific interaction. Methods: In this work, we demonstrate the potential of microstructure as an optical biosensor. Visible wavelength is utilized because it is commonly used in biological and chemical sensing for both label and label-free sensing. The SU8 polymer microstructures waveguides were fabricated on $3.5 \mu \mathrm{m}$ oxide. The microstructures are simulated using COMSOL Multiphysics. Simulation was recorded based on refractive index that mimic the bioanalytes solution and biological binding. Then the experimental setup is developed to control the optical component and manipulate the liquid samples. The fabricated devices were characterized by using the end-facet technique. Meanwhile, microfluidic channel system was also constructed in order to inject the liquid sample into the sensor surface. Results: The wavelength shift for microresonator structure approximately $41.2 \mathrm{~nm}$ for 0.1 increments of surrounding refractive index. The experiments demonstrate that the shift occurred approximately 22.5 $\mathrm{nm}$. In other hand, simulated Bragg grating structure gained the shift at $63.2 \mathrm{~nm}$. Meanwhile, the experimental results achieve approximately $20.3 \mathrm{~nm}$. Conclusions: Thus, both simulation and experimental results strongly indicate that patternable polymer microstructure has a huge potential for optical biosensing applications at visible region.

KEYWORDS: Optical biosensor, COMSOL Multiphysics, label-free sensing, polymer, visible light 